\title{
Growth of Single Crystal Tungsten Nanorods by Oblique Angle Sputter Deposition
}

Tansel Karabacak, Pei-I Wang, Gwo-Ching Wang, and Toh-Ming Lu

Department of Physics, Applied Physics, and Astronomy

Rensselaer Polytechnic Institute

Troy, NY 12180-3590

\section{ABSTRACT}

We report the creation of single crystal tungsten nanorods with unusual simple cubic $\beta$ phase. These novel nano-structures were grown by oblique angle sputter deposition with substrate rotation through a shadowing effect. Transmission electron microscopy (TEM) diffraction patterns from individual nanorods clearly show the single crystal structure. It is evident from TEM diffraction measurements, during the oblique angle deposition, both $\beta$-phase $\mathrm{W}(100)$ and $\alpha$-phase W(110) islands exist at the initial stages of growth. However, at later stages of the growth the $\beta$-phase structure dominates. This is in contrast to the sputter deposition at normal incidence where only the thermodynamically stable bcc $\alpha$-phase $W(110)$ polycrystalline films were formed when the film grew to a certain thickness. We explain our results by using the shadowing and adatom mobility mechanisms: At the initial stages of growth, the $\beta$-phase W(100) islands grow taller due to the lower adatom mobility on these islands. The taller $\beta$-phase W(100) islands survive in the competition during oblique angle growth and form isolated nanorods in the later stages, while the shorter $\alpha$-phase W(110) islands stop growing due to the shadowing effect. In addition, our Monte Carlo simulation results agree well with the experimental measurements.

\section{INTRODUCTION}

Tungsten films have been the subject of intense research due to their importance in various technological applications, for example, field emitters [1], photonic crystals [2], diffusion barriers in semiconductor interconnect structures [3], absorbing layers in X-ray masks [4], and X-ray mirrors [5]. Depending on the growth conditions and thickness of the films, normal incidence sputter deposition (NISD) of tungsten films can give rise to either the $\alpha$-phase W, which has the equilibrium bcc structure, or the metastable $\beta$-phase $\mathrm{W}$, which has an A15 (cubic) structure, or a mixture of both phases [6,7]. The lattice constants are $3.16 \AA$ and $5.04 \AA$ for $\alpha$ $\mathrm{W}$ and $\beta-\mathrm{W}$, respectively. These two phases may have very different properties, for example, the measured resistivity of $\beta-\mathrm{W}$ film is an order of magnitude higher than that of the $\alpha-\mathrm{W}$ film [3]. It was suggested that oxygen incorporation might play a role in the formation of the metastable $\beta$ W.

In a previous study [8] we reported a sequence of X-ray $\theta-2 \theta$ measurements for the NISD and oblique angle sputter deposited (OASD) tungsten films at different deposition times. It was realized that $\alpha$ - and $\beta$-phases are present at the initial stages of growth for both normal incidence and oblique angle deposition. As the growth progressed to a larger thickness the $\alpha$-phase for the NISD grew faster and the $\beta$-phase was suppressed. This behavior is consistent with that reported in the literature [7,9]. On the other hand, for the OASD we observed an opposite behavior. The $\beta$-phase grew faster as a function of time and the $\alpha$-phase almost disappeared. The final $\beta$-phase nanocolumns were stable against the transformation into the $\alpha-\mathrm{W}$ phase. We explained our 
results by a competitive growth mechanism between the crystal structures that had different adatom mobilities. During the OASD, $\beta$-phase islands of lower adatom mobility grew taller and could capture majority of incident atoms due to the shadowing effect. However, in order to demonstrate the predicted growth mechanism, detailed crystal phase information for the very early stages of growth, and a quantitative theoretical approach are needed.

In this paper, we present transmission electron microscopy (TEM) images and Monte Carlo simulations to explain the crystal structure evolution during the OASD and NISD. In addition, we show that, isolated $\beta-\mathrm{W}(100)$ nanorods formed during the OASD are single crystal. This can be an important property for various technological applications. For example, in a separate study we show that single crystal $\mathrm{W}$ nanorods by the OASD present superior field emission property [10].

\section{EXPERIMENTAL}

In our experiment, a dc magnetron sputtering system was used to deposit tungsten films. The films were deposited on oxidized p-Si(100) (resistivity $12-25 \Omega-\mathrm{cm})$ substrates $\left(\sim 2 \times 2 \mathrm{~cm}^{2}\right.$ size) using a $99.95 \%$ pure $\mathrm{W}$ cathode (diameter $\sim 7.6 \mathrm{~cm}$ ). The substrates were RCA cleaned and mounted on the sample holder located at a distance of $\sim 15 \mathrm{~cm}$ from the cathode. In the OASD [8], the substrate is tilted so that the angle between the surface normal of the target and the surface normal of the substrate can be large. In our present case, the angle was $87^{\circ}$. The substrate rotation speed was set to $0.5 \mathrm{~Hz}(30 \mathrm{rpm})$. In the NISD the tilt angle was set to $0^{\circ}$, with no sample rotation. The base pressure of $\sim 2 \times 10^{-6}$ Torr was achieved by a turbo-molecular pump backed by a mechanical pump. In all the deposition experiments, the power was 200 Watts at an ultra pure Ar pressure of 1.5 mTorr. The deposition rates were measured to be $\sim 8.5 \mathrm{~nm} / \mathrm{min}$ for the NISD and $\sim 5.0 \mathrm{~nm} / \mathrm{min}$ for the OASD. The thickness of the films was determined by a stepprofilometer and also verified by scanning electron microscopy (SEM) cross-sectional images. The maximum temperature of the substrate during the deposition was measured to be $\sim 85^{\circ} \mathrm{C}$.

Long ( $325 \mathrm{~nm})$ and individual $\mathrm{W}$ nanorods were removed from the Si substrate and then dispersed onto the lacy carbon film on a $\mathrm{Cu}$ grid for the TEM investigation. For the TEM study of crystal structure evolution at the early stages, thin $\mathrm{W}$ films (from $\sim 12 \mathrm{~nm}$ up to $\sim 50 \mathrm{~nm}$ ) were grown by the NISD and OASD directly on the carbon film placed on a $\mathrm{Cu}$ grid (from Ernest F. Fullam, Co). Then, the microstructure was studied using a Philips CM-12 microscope operating at $120 \mathrm{kV}$. In addition, selective area electron diffraction (SAED) was employed to characterize the crystal structure.

\section{$\underline{\text { Geometrical structure }}$}

Figures 1(a) and 1(b) show SEM cross sectional views of the final films deposited by NISD and OASD, respectively. For the NISD film, the cross section in Fig. 1(a) shows the commonly observed columnar structure that is a characteristic of a sputter deposited film. The columnar structure is a result of the shadowing effect due to the fact that not all incident flux arrive perpendicular to the surface. In fact, atoms obey a cosine distribution [11]. The shadowing effect leads to the columnar structure. The columns are not isolated and the film is continuous. On the other hand, the cross sectional view of the OASD film in Fig. 1(b) shows the structure of the isolated rods. 

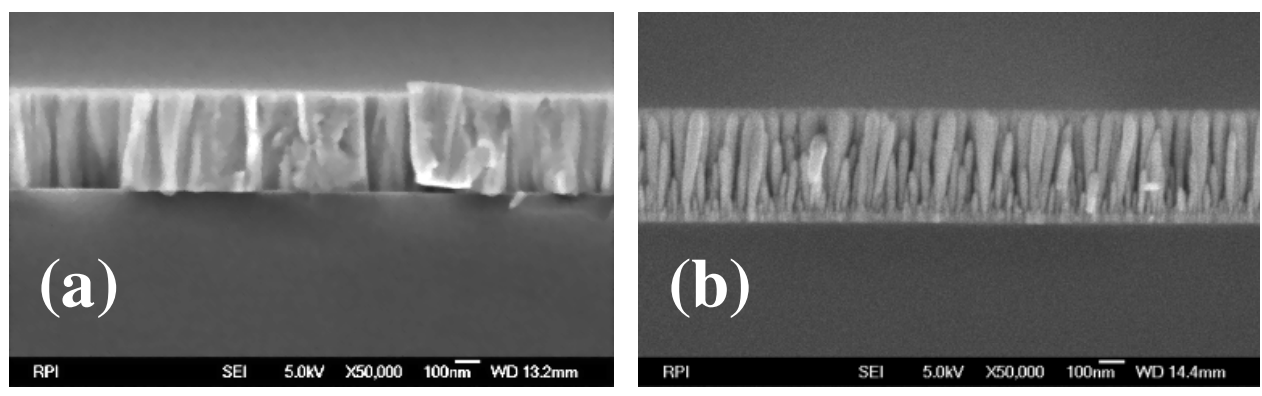

Figure 1. (a) Cross sectional SEM images of tungsten films made by the NISD (normal incidence sputter deposition) and (b) nanorods of tungsten made by the OASD (oblique angle sputter deposition) with substrate rotation.

\section{Single crystal nanorods}

It is evident from a previous X-ray diffraction (XRD) study [8] that $\beta$-W(100) phase was favored in $\mathrm{W}$ nanorods grown by the OASD. In addition, the pyramidal shape of nanorod tops with four-fold symmetric (110) facets suggested that the nanorods could be single crystals. However, the evidence that $\mathrm{W}$ nanorods are in fact single crystals comes from the TEM investigation of individual nanorods. Figure 2(a) shows the bright field TEM image of an individual $\mathrm{W}$ nanorod and its corresponding SAED pattern in Fig. 2(b). The existence of a single set of diffraction spots in the SAED pattern indicates the $\mathrm{W}$ nanorod is single crystal.

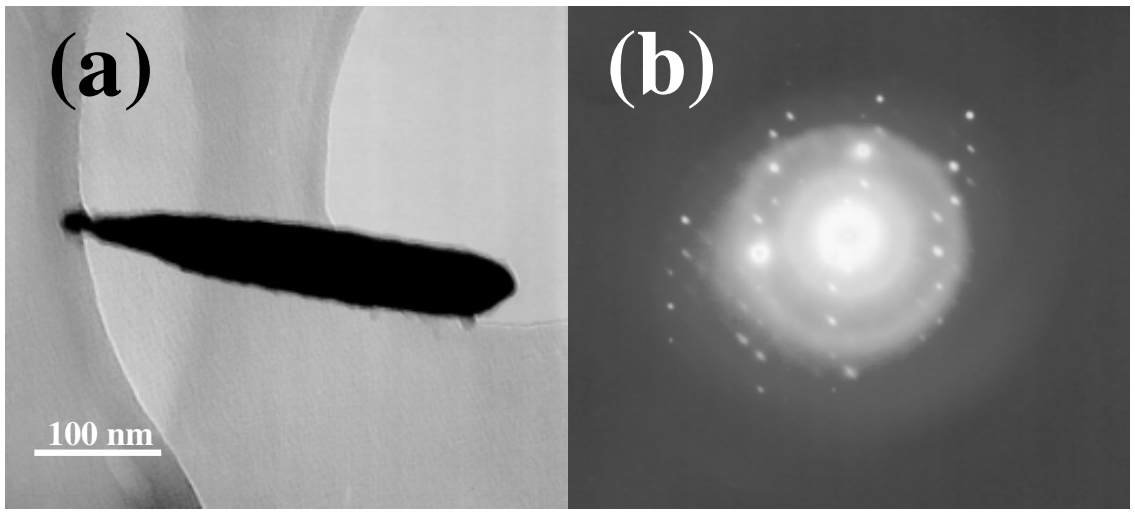

Figure 2. (a) Bright field TEM image of an individual $\mathrm{W}$ nanorod and (b) its corresponding SAED pattern (selective area electron diffraction). A single set of diffraction spots in the SAED pattern indicates that the $\mathrm{W}$ nanorod is a single crystal. The diffraction rings at the background in Fig. 2(b) originate from the underlying carbon film.

\section{Evolution of the crystal structure}

Figures 3(a)-3(1) show a sequence of bright field TEM images and corresponding SAED patterns of W films by the NISD (a-f) and W nanorods by the OASD ( $g-1)$ grown at various film thicknesses. The thickness values $(\sim 12-50 \mathrm{~nm})$ correspond to the initial stages of the deposition, which could not be resolved by the previous XRD study [8]. It is realized from Fig. 3 that both the $\alpha$ - and $\beta$-phases are present at the initial stages of growth for both the NISD and OASD. As the film grows to larger thickness the $\alpha$-phase for the normal incidence deposition grows faster and the $\beta$-phase is suppressed. In fact, the surface features that get larger in size with time as seen in the bright field images of the NISD films belong to the $\alpha$-phase. On the other hand, we 
see an opposite trend for the OASD nanorods. The $\beta$-phase grows faster as a function of time and the $\alpha$-phase starts to disappear. The formation of isolated $\beta$-phase islands during the OASD can also be realized as the film thickness increases (see Fig. 3(k)). Due to the shadowing effect, these islands serve as seeds to induce the subsequent growth of single crystal nanorods.

It was shown before that $\alpha-\mathrm{W}$ is the thermodynamically favorable state and the surface adatom mobility is higher than that on the $\beta$-phase surface [12]. The $\beta-\mathrm{W}$ is considered to be metastable with poor adatom mobility. Therefore, the reason why $\alpha-\mathrm{W}$ islands are laterally larger in Fig. 3 can be understood through the higher surface diffusion rates on these islands. In addition, at later stages their larger size enhances the probability of capturing freshly deposited atoms and diffusing adatoms. Eventually, the $\alpha-\mathrm{W}$ islands grow faster in the lateral directions in the substrate plane and cover up the $\beta-\mathrm{W}$ islands. On the other hand, during the oblique angle deposition, due to the extreme shadowing effect we expect that the islands that grow taller in the
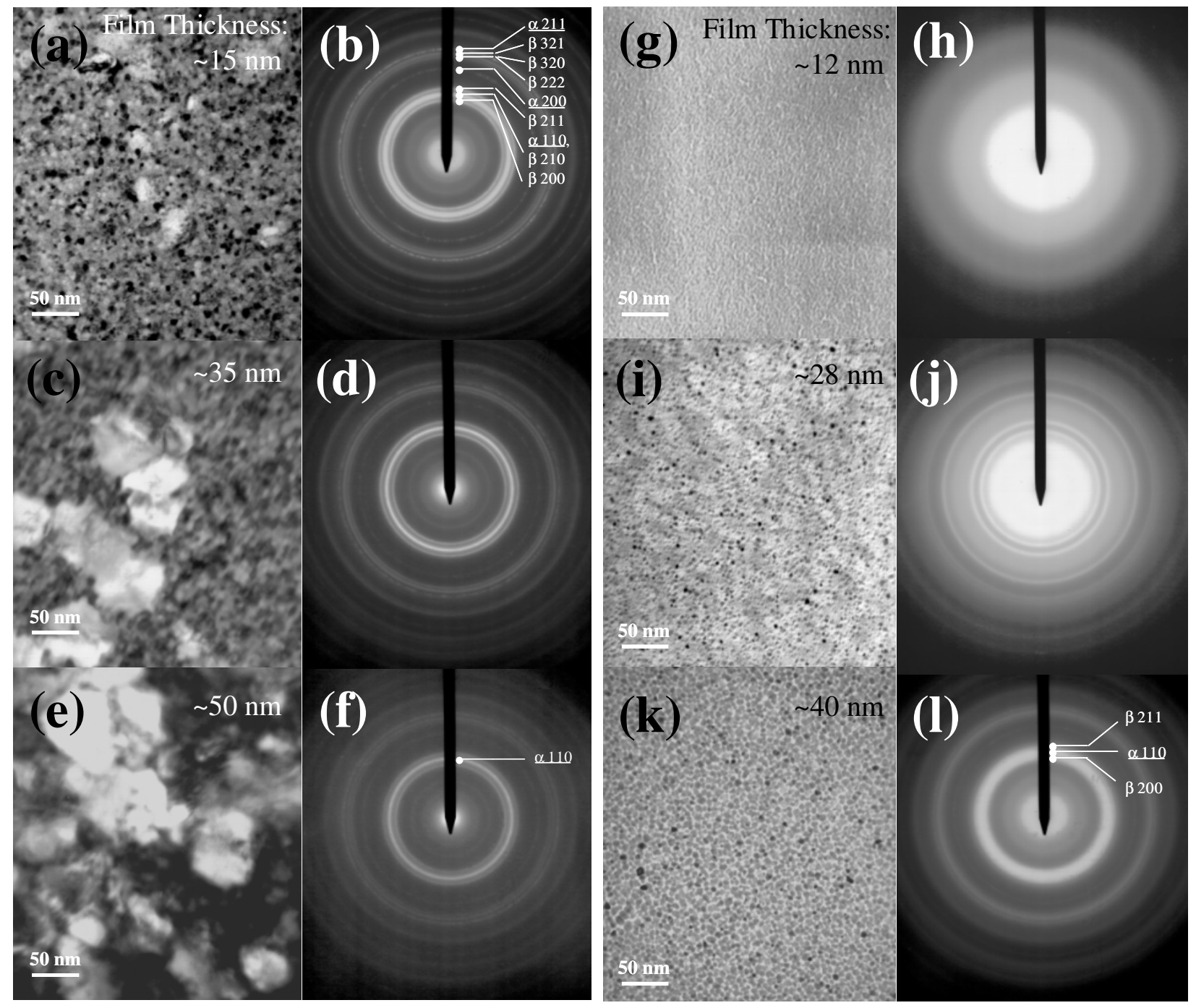

Figure 3. (a-f) Bright field TEM images and corresponding SAED patterns of the continuous $\mathrm{W}$ films by the NISD and (g-l) W nanorods by the OASD show the evolution of the microstructure during the early stages of growth. 
vertical direction will shadow a considerable amount of surface area in the film. Therefore, we expect a faster growth rate of the lower mobility $\beta-\mathrm{W}$ islands. The $\alpha-\mathrm{W}$ features are shadowed by the $\beta-\mathrm{W}$ rods and the growth of the $\alpha-\mathrm{W}$ is therefore suppressed.

\section{SIMULATIONS}

In order to understand this growth behavior we use a (2+1)-dimensional Monte Carlo method to simulate the evolution of morphological and crystal structure during normal incidence and oblique angle depositions. The details of the simulation method will be given elsewhere. Briefly, three-dimensional lattice is formed by cubic lattice points and each incident atom had the dimension of one lattice point. The simulations include an obliquely incident flux at angle $\theta$, substrate rotation, and surface diffusion. At each simulation step an atom is sent towards a randomly chosen lattice point on the surface of size $L \times L$. To take into account the substrate rotation, each atom is sent with a change in the azimuthal angle of $\Delta \phi$ degrees from the previous one. After the incident atom is deposited onto the surface, an atom that is chosen randomly on the surface is set to diffuse to another nearest neighbor random location. The probability of diffusion is proportional to $\exp \left(-E / k_{b} T\right)$, where $E$ is the sum of activation energy for diffusion $\left(E_{D}\right)$ and total bond energies with nearest neighbors (i.e. $E_{n}$ for single bond and $n n E_{n}$ for $n n$ number of nearest neighbors). $k_{b}$ stands for the Boltzmann constant and $T$ is the substrate temperature in Kelvins. To study the crystal structure evolution we randomly label the incident atom as being either $\alpha$ or $\beta$. Therefore, if the particle is deposited on the substrate it forms a nucleation site for $\alpha$ or $\beta$ phase with equal probability. However, if it is deposited on an existing island with a specific phase of $\alpha$ or $\beta$ then its type is set to this island's phase. For the diffusion process we assign different activation and bond energies for $\alpha, \beta$, and substrate sites. This mimics the effect of different adatom mobilities that depend on the type of the crystal phase. In cases where a diffusing adatom moves on top of an island of different phase, the type of the adatom is changed only if the $n n$ of the other phase outnumbers the $n n$ having the same phase with the adatom.

Figure 4 shows an example profile for the simulated volume ratios of $\alpha$ - and $\beta$-phases after we assigned high adatom mobilities (i.e. low $E_{D}$ and $E_{n}$ ) to $\alpha$-phase surface sites, and low adatom mobilities to the $\beta$-phase. It is realized that during normal incidence deposition the film structure becomes dominated by the $\alpha$-phase as the thickness increases. On the other hand, during the oblique angle growth, the $\beta$-phase nanorods of low adatom mobility can grow faster in height. Incident particles preferentially deposit on these nanorods due to the shadowing effect and this leads to the evolution of a dominant $\beta$-phase. Therefore, our simulation results agree well with our experimental measurements.

\section{CONCLUSIONS}

In conclusion, we presented detailed experimental and simulation studies to investigate the evolution of crystal structure during the oblique angle and normal incidence sputter depositions of $\mathrm{W}$ films. We were able to obtain single crystal $\beta-\mathrm{W}(100)$ nanorods by the oblique angle sputter deposition technique. We explain the formation of $\beta$-phase nanorods by a combination of a shadowing effect and the difference in the adatom surface mobilities between different crystal planes. Our simulation results are consistent with the experimental measurements.

\section{ACKNOWLEDGEMENTS}

Work supported by NSF and Harry Meiners Fellowship. 


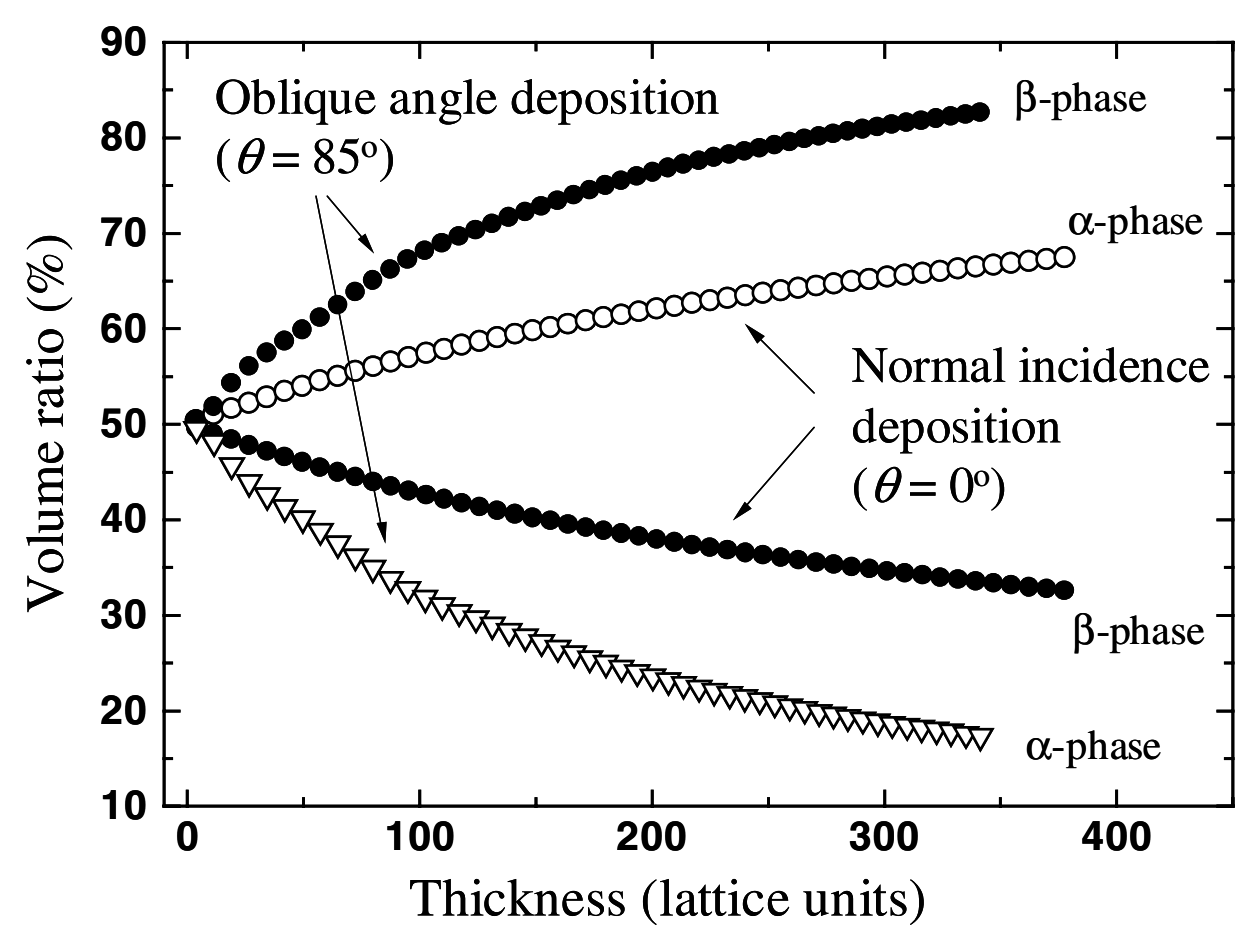

Figure 4. Evolution of simulated volume ratios of $\alpha$ - and $\beta$-phase film sites during the oblique angle and normal incidence depositions are shown. The simulation parameters are the same for both the NISD and OASD except the deposition angle, where $\theta=0^{\circ}$ for the NISD and $\theta=85^{\circ}$ for the OASD. Adatom mobilities are set to be higher on $\alpha$-phase islands compared to $\beta$-phase islands. Common simulation parameters are $L \times L=512 \times 512, \Delta \phi=0.036^{\circ}, T=358^{\circ} \mathrm{K}$, and $E_{D}=$ $E_{n}=0.05 \mathrm{eV},=0.1 \mathrm{eV}$, and $=0.5 \mathrm{eV}$ on $\alpha$-phase, $\beta$-phase, substrate surface sites, respectively.

\section{REFERENCES}

1. C.-H. Choi, Y.-T. Jang, B.-K. Ju, Y.-H. Lee, M.-H. Oh, J.-H. Ahn, and N.-K. Min, SID Digest 3301, 369 (2002).

2. J. G. Fleming, S. Y. Lin, I. El-Kady, R. Biswas, and K. M. Ho, Nature 417, 52 (2002).

3. S. M. Rossnagel, I. C. Noyan, and C. Cabral Jr., J. Vac. Sci. Technol. 20, 2047 (2002).

4. M. Itoh, M. Hori, and S. Nadahara, J. Vac. Sci. Technol. B 9, 149 (1991).

5. M. S. Aouadi, R. R. Parsons, P. C. Wong, and K. A. R. Mitchell, J. Vac. Sci. Technol. A 10, 273 (1992).

6. Y. G. Shen and Y. M. Mai, Mater. Sci. Eng. A 28, 176 (2000).

7. I. A. Weerasekera, S. I. Shah, D. V. Baxter, and K. M. Unruh, Appl. Phys. Lett. 64, 3231 (1994).

8. T. Karabacak, A. Mallikarjunan, J. P. Singh, D.-X. Ye, G.-C. Wang, and T.-M. Lu, Appl. Phys. Lett. 83, 3096 (2003).

9. I. C. Noyan, T. M. Shaw, and C. C. Goldsmith, J. Appl. Phys. 82, 4300 (1997).

10. J. P. Singh, F. Tang, T. Karabacak, T.-M. Lu, and G.-C. Wang, submitted.

11. D. L. Smith, Thin-Film Deposition: Principles and Practice (McGraw-Hill, New York, 1995), p. 96 and p. 432.

12. Y. G. Shen, Y. W. Mai, Q. C. Zhang, D. R. McKenzie, W. D. McFall, and W. E. McBride, J. Appl. Phys. 87, 177 (2000). 